\title{
RecQ helicase BLM regulates prostate cancer cell proliferation and apoptosis
}

\author{
XIAOSONG QIAN, SUJUAN FENG, DAWEI XIE, DALIN FENG, YIHANG JIANG and XIAODONG ZHANG \\ Institute of Uro-Nephrology, Beijing Chao-Yang Hospital, Capital Medical University, Beijing 100020, P.R. China
}

Received January 15, 2017; Accepted July 11, 2017

DOI: $10.3892 / 01.2017 .6704$

\begin{abstract}
Prostate cancer (PCa) is a common malignant tumor and the second leading cause of morbidity and mortality in men worldwide. Considering the prevalence and effects of $\mathrm{PCa}$ in males, an understanding of the molecular mechanisms underlying PCa tumorigenesis are essential and may provide novel therapeutic strategies for treating $\mathrm{PCa}$. Bloom syndrome protein (BLM) is a member of the RecQ helicase family. The major function of BLM is to uncoil the double-stranded DNA structure. It has previously been demonstrated that BLM acts as a 'genome caretaker', and dysregulation of BLM function has been implicated in the development of multiple tumor types; however, its potential for inducing PCa tumorigenesis remains undetermined. The present study aimed to explore the function of BLM in PCa progression. Reverse transcription-polymerase chain reaction, immunohistochemistry and western blot analyses were performed to detect the BLM expression pattern in $\mathrm{PCa}$ patients and cell lines. The proliferation, and migration and invasion capacities of prostate cells were determined by EdU and Transwell assays following transfection with BLM-targeting short hairpin RNA (shRNA). The expression of BLM was significantly increased in PCa tissues and PC3 cells compared with non-PCa tissues and benign prostatic hyperplasia cells Knockdown of BLM via shRNA inhibited PCa cell proliferation, and promoted PCa cell apoptosis. Notably, reducing the expression of BLM had no effect on the migration or invasive abilities of PCa cells. These results suggest that downregulation of BLM may alleviate $\mathrm{PCa}$ development, providing a novel perspective for $\mathrm{PCa}$ tumorigenesis and a potential therapeutic target for $\mathrm{PCa}$.
\end{abstract}

\section{Introduction}

Prostate cancer (PCa) is a common malignant tumor and the second leading cause of male morbidity and mortality worldwide. In 2015, PCa alone accounted for 26\% $(220,800)$

Correspondence to: $\mathrm{Dr}$ Xiaodong Zhang, Institute of Uro-Nephrology, Beijing Chao-Yang Hospital, Capital Medical University, 8 Gong Ti Nan Lu, Beijing 100020, P.R. China

E-mail: drcyzxd@163.com

Key words: apoptosis, bloom syndrome protein, RecQ, proliferation, prostate cancer of newly diagnosed cancer cases in men and led to $>27,000$ deaths (1). Although numerous methods, such as biochemical assays (2), biopsies (3), digital rectal examination (DRE) (4), and transrectal ultrasonography (5), have been widely used in PCa diagnosis, lack of early efficient diagnoses is the major problem affecting precise therapy of PCa. Therefore, research on the mechanisms of PCa tumorigenesis and progression will help to improve prevention strategies and develop effective interventions to treat $\mathrm{PCa}$.

Bloom syndrome protein (BLM), which is mutated in patients with Bloom syndrome, is a member of the RecQ helicase family. The core structure is highly conserved between yeast and humans (6). Usually, BLM utilizes energy from ATP-hydrolysis to unwind double-stranded DNA (dsDNA) with $3^{\prime}$ to 5' polarity, and to disassemble complex DNA structures, such as G-quadruplexes (G4s) and Holliday junctions (HJs) that are formed during the $S$ phase of the cell cycle (7). BLM is a crucial helicase required for DNA metabolic processes, including DNA recombination, replication and repair. Such helicases are referred to as 'the caretakers of the genome'. BLM is expressed at high levels in tumor cells, and its level expression is known to be differentially regulated through the cell cycle stages. Considering that abnormalities in BLM are linked to genome instability, accumulating evidence has recently indicated that changes in BLM protein expression are associated with the development of a variety of tumors (8-10). Using exome sequencing and gene analysis, Johnson et al (11) identified that BLM missense variants and PCa status were completely co-segregated. However, the role of BLM in PCa, and whether it can influence the cell cycle and apoptosis, still remains unclear. The results of the present study demonstrate that BLM protein expression is upregulated in PCa patient tissues and PC3 cells. Furthermore, the downregulation of BLM can inhibit PC3 cell ischemic proliferation and induce apoptosis in vitro.

\section{Materials and methods}

Tissue collection. A total of 15 cancerous prostate tissues (PCa) and 10 noncancerous tissues (non-PCa) were collected from patients who underwent surgery at Beijing Chao-Yang Hospital, Capital Medical University (Beijing, China). The present study was approved by the ethics committee of Beijing Chao-Yang Hospital, Capital Medical University, and written informed consent was obtained from each patient. 
Immunohistochemistry. All tissues from PCa patients were paraffin-embedded and cut to $4 \mathrm{~mm}$ thickness. Endogenous peroxidase activity in deparaffinized sections was blocked by incubation with $3 \%$ hydrogen peroxide. Subsequently, sections were boiled in $1 \mathrm{mM}$ EDTA $\mathrm{pH} 8.0$ for $10 \mathrm{~min}$, followed by cooling at room temperature. Fetal bovine serum was applied to block non-specific antigen sites. Sections were incubated with anti-BLM antibody for $3 \mathrm{~h}$ at a dilution of 1:200, and then the secondary antibody was applied for $30 \mathrm{~min}$. Finally, the sections were stained with liquid diaminobenzydine and counterstained with Mayer's hematoxylin blue.

Cells and culture. The BPH1 benign prostatic hyperplasia cell line and PC3 PCa cell line were obtained from the American Type Culture Collection (Manassas, VA, USA). Cells were routinely cultured in RPMI-1640 medium (Gibco; Thermo Fisher Scientific, Inc., Waltham, MA, USA), supplemented with $10 \%$ fetal bovine serum (Gibco) in a standard culture conditions $\left(5 \% \mathrm{CO}_{2}\right.$ at $\left.37^{\circ} \mathrm{C}\right)$.

BLM short hairpin RNA (shRNA) plasmid transfection. PC3 cells were transfected with BLM-targeting shRNA (GeneChem Co. Ltd, Shanghai, China) (target sequence: AAGGAAGTT GTATGCACTA) or non-targeting shRNA (GeneChem Co. Ltd, Shanghai, China) using Lipofectamine 2000. Transfection with a negative control (NC) scramble sequence was also conducted. Transfection efficiency was confirmed by reverse transcription-quantitative polymerase chain reaction (RT-qPCR) and western blot.

Cell proliferation assay. PC3 cells (1x103) were seeded in 6 -well plates in RPMI-1640 containing 10\% FBS. Cells were harvested and counted using the EdU assay after 2, 4 and 7 days, as follows. Cell proliferation was detected by using the Cell-LightTM 5-ethynyl-2'-deoxyuridine (EdU) imaging detection kit according to the manufacturer's instructions (RiboBio, Guangzhou, China). EdU was added to cells seeded in 6-well plates and the cells were cultured for an additional $2 \mathrm{~h}$. The cells were then collected and washed with PBS twice. Apollo reaction buffer was then incubated with the cells for $10 \mathrm{~min}$ in the dark. Subsequently, cells were washed once with PBS, stained with Hoechst 33342 for $30 \mathrm{~min}$ at room temperature and washed with PBS again. EdU-positive cells were counted using Image-Pro Plus 6.0 software (Media Cybernetics, Silver Springs, MD, USA) in five randomly selected fields.

For cell cycle analysis, cells were seeded on 6-well plates and stained with Vybrant ${ }^{\circledR}$ DyeCycle ${ }^{\mathrm{TM}}$ Ruby stain kit at $37^{\circ} \mathrm{C}$ for $30 \mathrm{~min}$. The DNA content was measured by flow cytometry according to the manufacturer's instructions (Thermo Fisher Scientific, Inc.).

Cell invasion and migration assays. A cell invasion assay was performed using a 24-well Transwell (Corning, New York, NY, USA) with a polycarbonate filter pre-coated with Matrigel at a 1:20 dilution (BD Biosciences, Franklin Lakes, NJ, USA). Cells $\left(2.5 \times 10^{4}\right)$ suspended in $0.2 \mathrm{ml}$ serum-free medium were added to the upper well of the chamber, and $500 \mathrm{ml}$ RPMI-1640 supplemented with $10 \%$ FBS was added to the lower well. After $24 \mathrm{~h}$ incubation, cells remaining in the upper well were removed by scraping. Invaded cells on the bottom
Table I. Clinical and pathological parameters of $\mathrm{PCa}$ and Non-PCa patients.

\begin{tabular}{lcc}
\hline Parameter & PCa $(\mathrm{n}=15)$ & Non-PCa $(\mathrm{n}=10)$ \\
\hline Age & & \\
Range & $58-76$ & $49-76$ \\
Mean & 68.40 & 64.60 \\
Total PSA & & \\
Range & $0.005-75.488$ & $1.550-10.383$ \\
Mean & 17.400 & 5.126 \\
Clinical stage & & \\
T1c & 2 & N/A \\
T2c & 5 & N/A \\
T3a & 2 & N/A \\
T3b & 4 & N/A \\
T4b & 2 & N/A \\
Gleason score & & \\
$3+3$ & 1 & N/A \\
$3+4$ & 2 & N/A \\
$4+3$ & 4 & N/A \\
$4+4$ & 5 & N/A \\
$4+5$ & 2 & N/A \\
$5+4$ & 1 & N/A \\
\hline
\end{tabular}

of the membrane were fixed with $4 \%$ paraformaldehyde and stained with $0.1 \%$ crystal violet solution. Cells were imaged under a microscope, and the cell numbers were counted using Image-Pro Plus 6.0 software (Media Cybernetics, Silver Springs, MD, USA) in five randomly selected fields. A cell migration assay was conducted in a similar manner but without the use of Matrigel.

Apoptosis assay. Cell apoptosis was detected using an Annexin V-PE apoptosis detection kit (BD Pharmingen, San Diego, CA, USA) according to the manufacturer's protocol. Briefly, cells at density of $1 \times 10^{6}$ cells $/ \mathrm{ml}$ were harvested and washed twice with PBS, then $500 \mu 1$ of cell suspension in binding buffer was transferred to a 5-ml falcon tube, and $5 \mu 1$ Annexin V-PE conjugate and $5 \mu \mathrm{l}$ propidium iodide were added. The cells were incubated in the dark for $15 \mathrm{~min}$ at room temperature and the fluorescence of the cells was subsequently determined by flow cytometry.

$R N A$ extraction and $R T-q P C R$. Total RNA was extracted from tissues or cells using TRIzol ${ }^{\circledR}$ reagent (Invitrogen; Thermo Fisher Scientific, Inc.). A FastQuant RT Kit (With gDNase) and SuperReal PreMix Plus (SYBR Green; TIANGEN BIOTECH, Beijing, China) were used. The PCR primers used were as follows: BLM, forward 5'-GGATCCTGGTTCCGTCCGC-3', reverse 5'-CCTCAGTCAAATCTATTTGCTCG-3'; $\beta$-actin, forward 5'-TGACGTGGACATCCGCAAAG-3', reverse 5'-TCTTCATTGTGCTGGGTGCC-3'. PCR cycling conditions were as follows: Initial denaturation period at $95^{\circ} \mathrm{C}$ for $15 \mathrm{~min}$, followed by a two-step PCR program consisting of denaturation at $95^{\circ} \mathrm{C}$ for $10 \mathrm{sec}$ and annealing/extension at $60^{\circ} \mathrm{C}$ for $32 \mathrm{sec}$ 
A

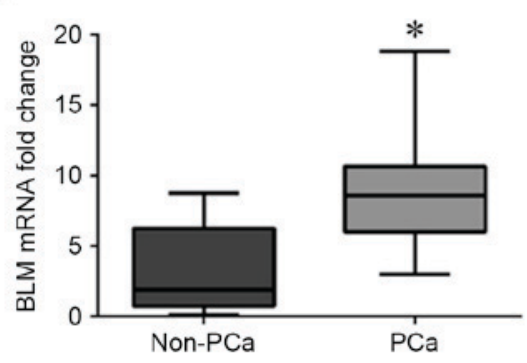

C

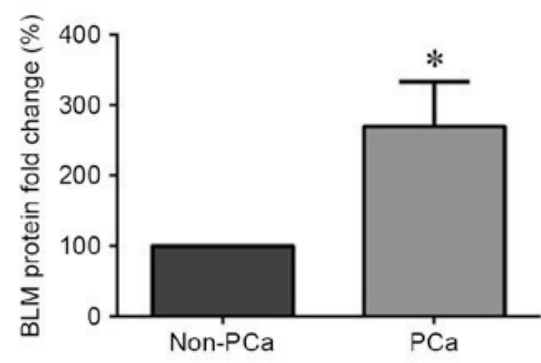

B

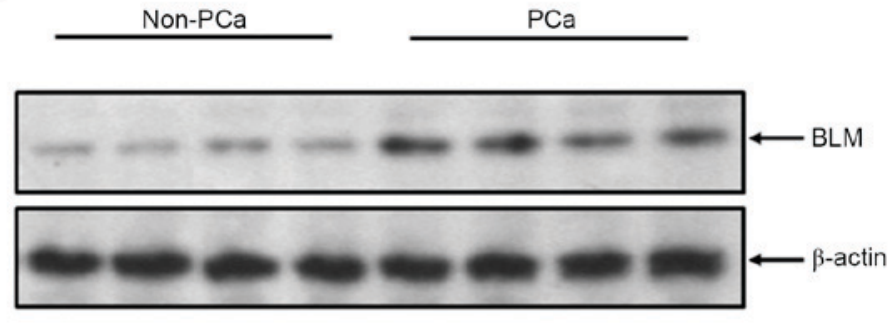

D

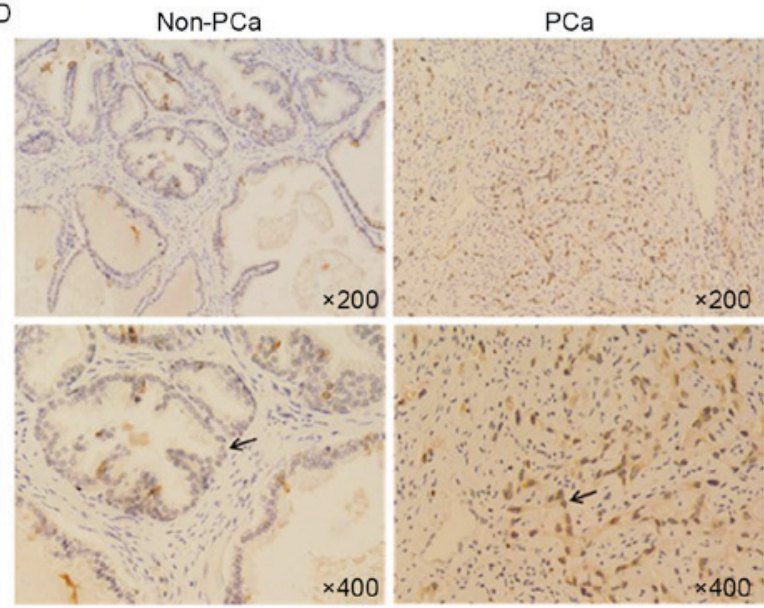

Figure 1. Higher expression of BLM in PCa patients. (A) The relative expression of BLM mRNA in PCa tissues was measured by qRT-PCR. $\beta$-actin acted as an internal loading control. Each sample was detected by triplicates, ${ }^{*} \mathrm{P}<0.05$; (B) Typical results of western blot analysis shows BLM protein level in PCa and non-PCa tissues. (C) The quantitative analysis demonstrates that the BLM protein level increases significantly in PCa tissues. ${ }^{*} \mathrm{P}<0.05$; (D) Immunohistochemical staining shows that the BLM is strong positive in the nuclear of prostate tissues (arrows) and significantly increases in PCa tissues compared to the non-PCa tissues. BLM, bloom syndrome protein; PCa, prostate cancer.

for 40 cycles. All samples were normalized against the internal control $\left(\beta\right.$-actin) and analyzed using the $2^{-\Delta \Delta C t}$ method.

Western blot analysis. Total protein was extracted from the cells or tissues using lysis buffer containing protease inhibitors. Proteins $(30 \mu \mathrm{g})$ from each sample were loaded on $10 \%$ SDS-polyacrylamide gels for electrophoresis. Following electrophoresis, the proteins were transferred onto polyvinylidene difluoride membranes (GE Healthcare, Chalfont, UK), which were then blocked with $10 \%$ non-fat milk in Tween/Tris-buffered salt solution (TTBS; $20 \mathrm{~mm}$ Tris-Cl, pH 7.5, $0.15 \mathrm{M} \mathrm{NaCl}$ and $0.05 \%$ Tween-20) for $1 \mathrm{~h}$. Following incubation with the anti-BLM antibody (Santa Cruz Biotechnology, Inc., Dallas, TX, USA) at $4^{\circ} \mathrm{C}$ overnight, the membrane was washed and incubated with IRDye ${ }^{\circledR} 800 \mathrm{CW}$ donkey anti-goat IgG secondary antibody at room temperature for $2 \mathrm{~h}$ followed by LI-COR Odyssey gel imaging scanner detection (LI-COR Biosciences, Lincoln, NE, USA). To verify equal loading of protein, the blots were reprobed with primary monoclonal antibody against $\beta$-actin (ProteinTech Group, Inc., Chicago, IL, USA).

Statistical analysis. Quantitative analysis of immunoblotting was performed using Quantity-One software (Gel Doc 2000 imaging system, Bio-Rad Laboratories, Inc., Hercules, CA, USA). For protein level, the protein ratio of BLM (band density of protein/band density of $\beta$-actin) was set as $100 \%$ in the BPH or NC group. The data from other groups were expressed as a percentage of the BPH or NC group. The values are presented as the mean \pm standard error. Statistical analysis was conducted by one-way analysis of variance followed by all pairwise multiple comparison procedures using the Bonferroni test and Student's t-test. $\mathrm{P}<0.05$ was considered to indicate a statistically significant difference.

\section{Results}

Differential expression of BLM in PCa and non-PCa patients. To determine BLM expression status in PCa tissues, the mRNA and protein levels of BLM in PCa and non-PCa tissue samples were detected. The patient profiles are summarized in Table I. We found that the BLM mRNA and protein expression levels were upregulated in the PCa tissues compared the non-PCa group (Fig. 1A-C). Similarly, immunohistochemical staining showed that the expression of BLM protein was increased in the nuclei of tumor cells, but it almost was undetectable in non-PCa tissue (Fig. 1D).

Changes of BLM expression level in PCa and BPH1 cells. To verify the results from tissue samples, the BLM expression level was also analyzed in BPH1 and PC3 cells in vitro. As shown in Fig. 2, the mRNA and protein levels of BLM in PC3 cells were significantly higher than that of the BPH1 cells.

Inhibition of BLM reduced PC3 cell proliferation in vitro. To explore the role of BLM in PCa cell proliferation, $\mathrm{PC} 3$ cells were transfected with a BLM-targeting shRNA plasmid. As shown in 
A

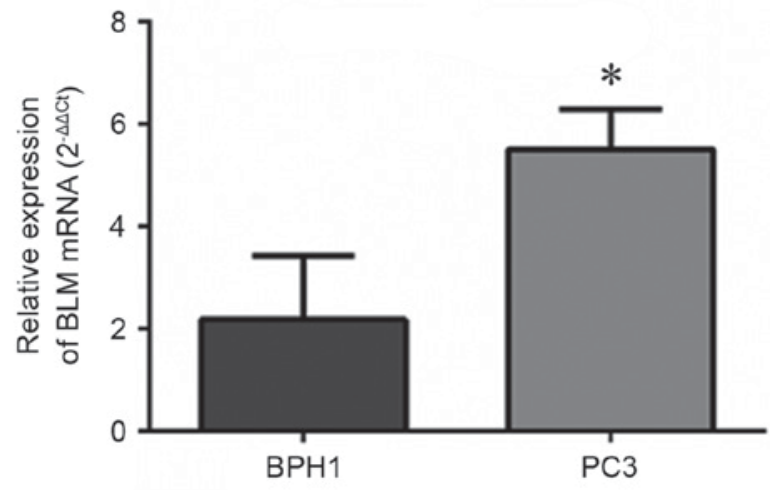

B
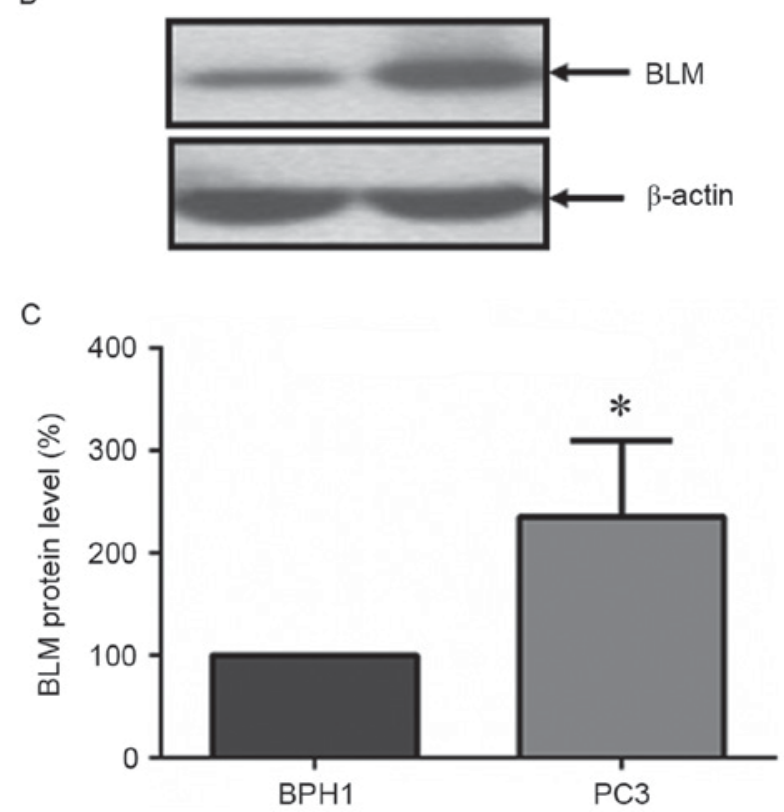

Figure 2. Increase expression of BLM in PC3 cells. (A) Relative expression of BLM mRNA is higher in PC 3 cells than in BPH1 cells, $n=3$ per group, ${ }^{*} \mathrm{P}<0.05$; (B) Western blot analysis shows that BLM protein level higher in PC 3 cells than BPH1 cells. (C) The quantitative analysis also demonstrates that the BLM protein level increases significantly in PC 3 cells, $n=3$ per group, ${ }^{*} \mathrm{P}<0.05$. BLM, bloom syndrome protein.

Fig. 3, both of the BLM mRNA and protein expression levels were significantly downregulated in PC3 cells after transfection with the BLM-targeting shRNA. An EdU proliferation assay was conducted to determine the effect of BLM inhibition on PC3 cell proliferation. As shown in Fig. 4A and B, shRNA-mediated inhibition of BLM significantly reduced PC3 cell proliferation. Additionally, cell cycle analysis was performed to examine if BLM inhibition influences the cell cycle. The results in Fig. 5A and B revealed that there was significant decrease in the number of cells in S-phase after transfection with BLM shRNA. Similarly, the PC3 cells exhibited a significantly reduced proliferative capacity when BLM was knocked down (Fig. 5D).

Downregulation of BLM promoted PCa cell apoptosis. To further elucidate the role of BLM in PCa apoptosis, PC3 cells transfected with BLM shRNA plasmid were subjected to Annexin-V and PI staining, and flow cytometry analysis was performed. The results shown in Fig. 6A and B demonstrated that knockdown of BLM led to an increase in apoptosis.
A

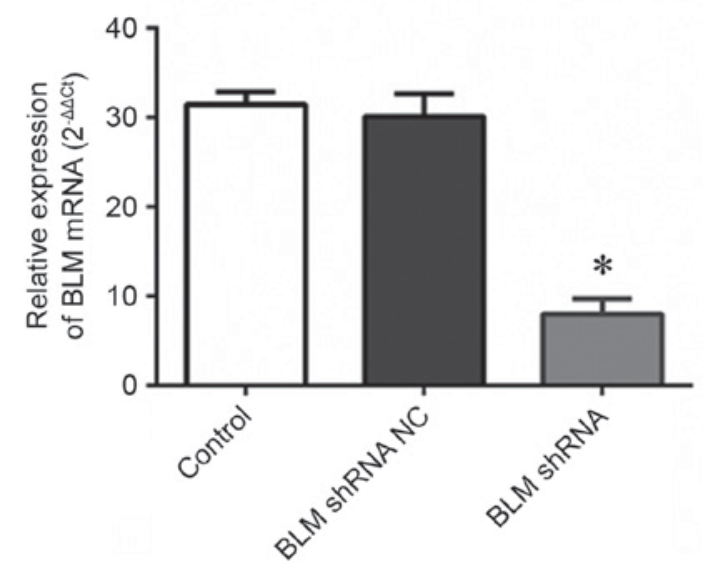

B

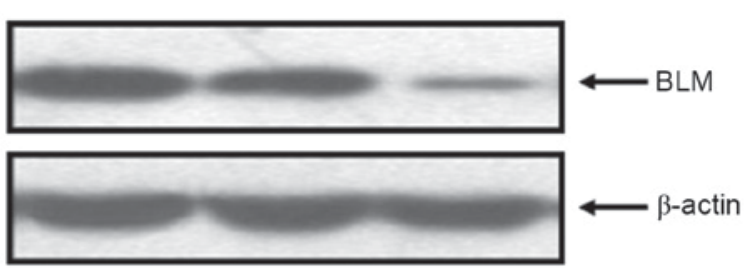

C

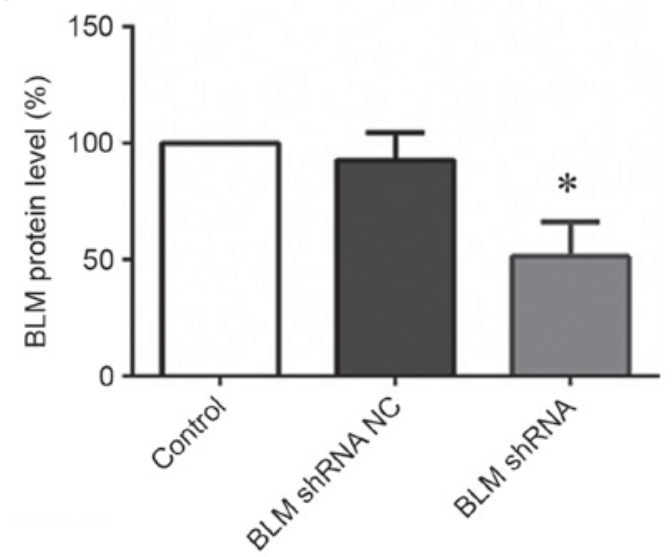

Figure 3. Effect of BLM shRNA on the BLM expression in PC3 cells (A) BLM mRNA level could be downregulated in BLM shRNA transfected PC 3 cells, $n=3$ per group, ${ }^{*} \mathrm{P}<0.05$. (B) Western blot analysis shows that BLM protein level significantly decreases in the BLM shRNA transfected PC3 cells than shRNA NC transfected PC 3 cells. (C) The quantitative analysis shows that the BLM protein level decreases significantly in PC 3 cells, $n=3$ per group, ${ }^{*} \mathrm{P}<0.05$. BLM, bloom syndrome protein.

Inhibition of BLM had no effect on PCa cell migration and invasion. The effect of BLM on PCa cell migration and invasion was examined using Transwell assays. As presented in Fig. 7A and B, PC3 cells transfected with the BLM shRNA plasmid did not exhibit any increase or decrease in migration and invasion compared with the NC cells.

\section{Discussion}

It is well-established that PCa development is affected by genetic inheritance, with numerous genes recognized to influence PCa susceptibility, including MED12 (12), HOXB13 (13), BRCA1 and BRCA2 (14,15). As a RecQ helicase, BLM is part 
A

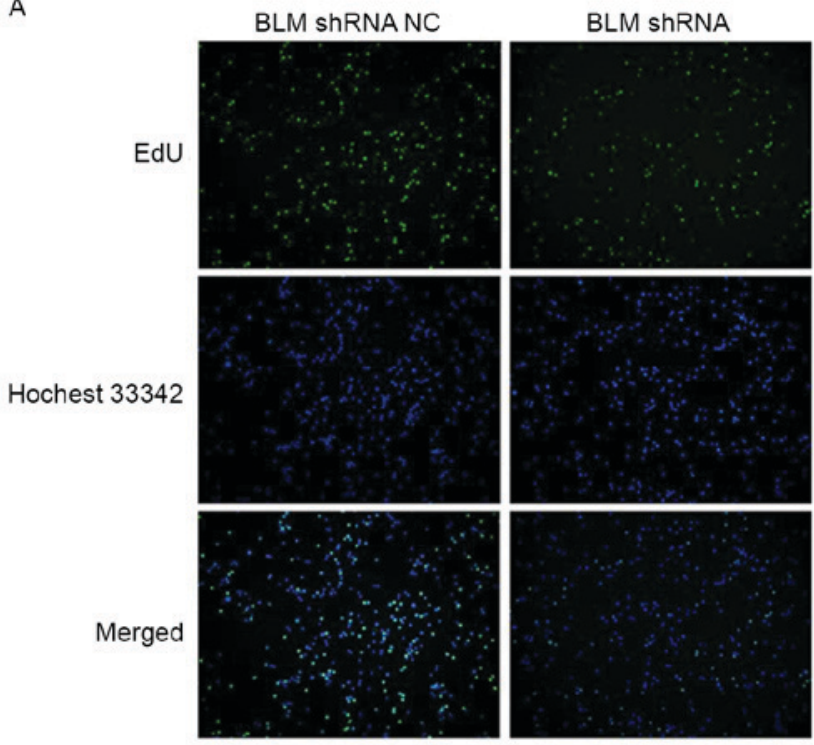

B

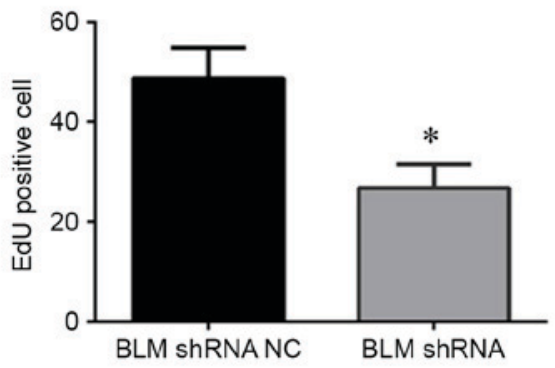

Figure 4. Knockdown of BLM decreases cell proliferation. (A) Representative results of images show the effect of BLM shRNA on PC3 cells proliferation. (B) The quantitative analysis of EdU positive cell number is decreased in PC3 cells transfected with BLM shRNA compared with the NC group, $n=3$ per group, ${ }^{*} \mathrm{P}<0.05$. BLM, bloom syndrome protein; NC, negative control.

A

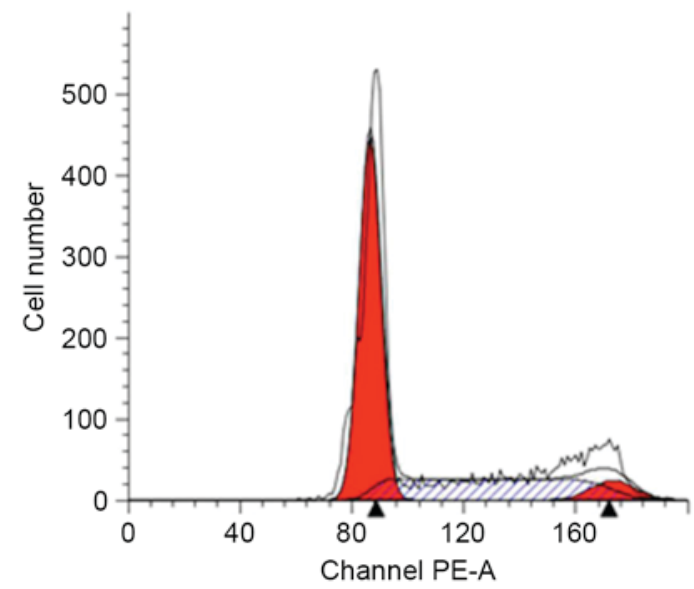

C

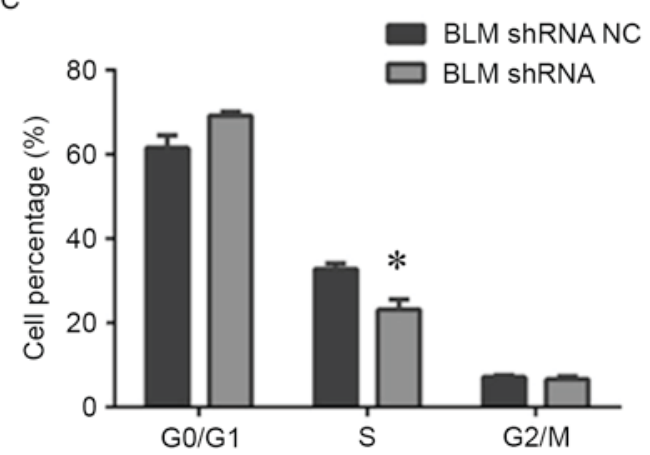

B

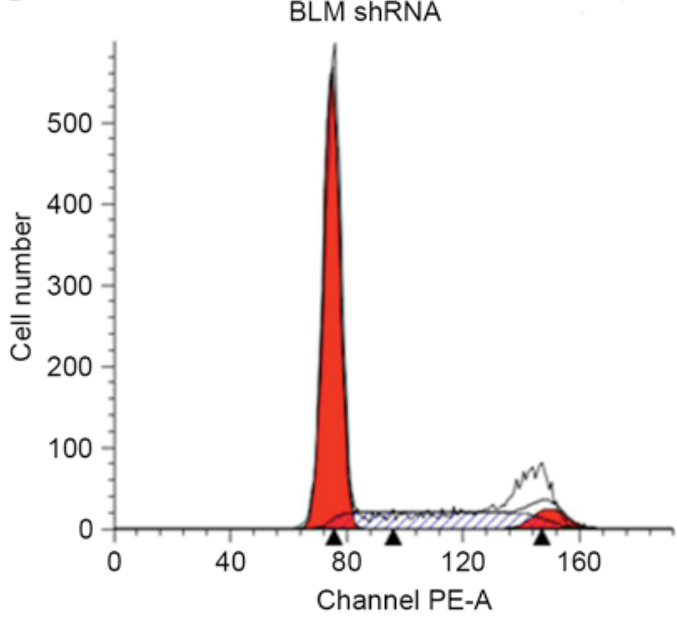

D

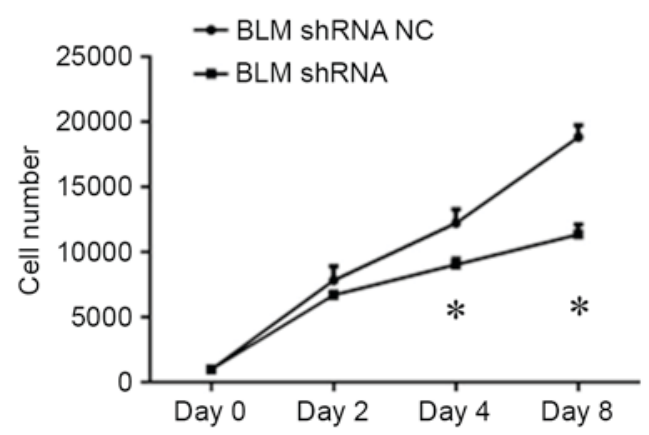

Figure 5. Knockdown of BLM induces cell cycle arrest. (A and B) Typical images of cell cycle progression were measured by flow cytometry. (C) The quantitative analysis demonstrated that knockdown of BLM decreases the number of cells in the S-phase, $n=3$ per group, ${ }^{*} \mathrm{P}<0.05$. (D) Cell counting decreases in PC3 cells transfected with BLM shRNA after 4 and 8 days seeded compared with the NC group, $\mathrm{n}=3$ per group, " $\mathrm{P}<0.05$. BLM, bloom syndrome protein.

of a family of DNA unwinding enzymes, and has crucial roles at multiple steps in the DNA recombination, replication and repair processes (16). The human genome contains five RecQ genes that encode Werner syndrome protein (WRN), BLM, RECQ1, RECQ4, and RECQ5. It is worth noting that BLM is mutated in Bloom syndrome, a rare autosomal recessive disorder that 
A

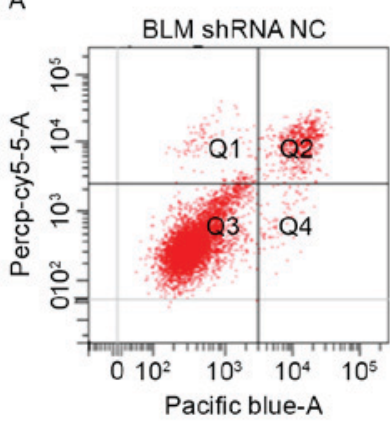

B

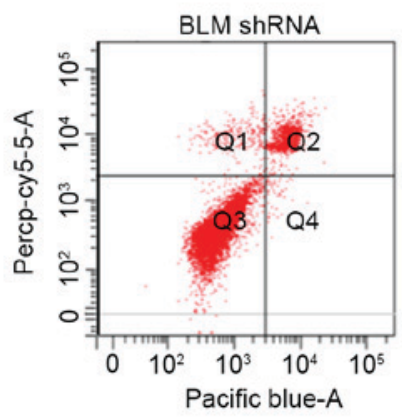

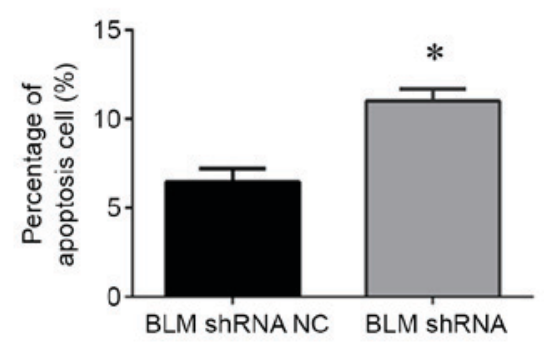

Figure 6. Downregulation of BLM promotes cell apoptosis. (A) Representative images of cell apoptosis were measured by flow cytometry. (B) Quantification of apoptotic cell number indicated that cells transfected with BLM shRNA enhanced PC 3 cell apoptosis compared with the NC group, $\mathrm{n}=3$ per group, " $\mathrm{P}<0.05$. $\mathrm{BLM}$, bloom syndrome protein; NC, negative control.

A

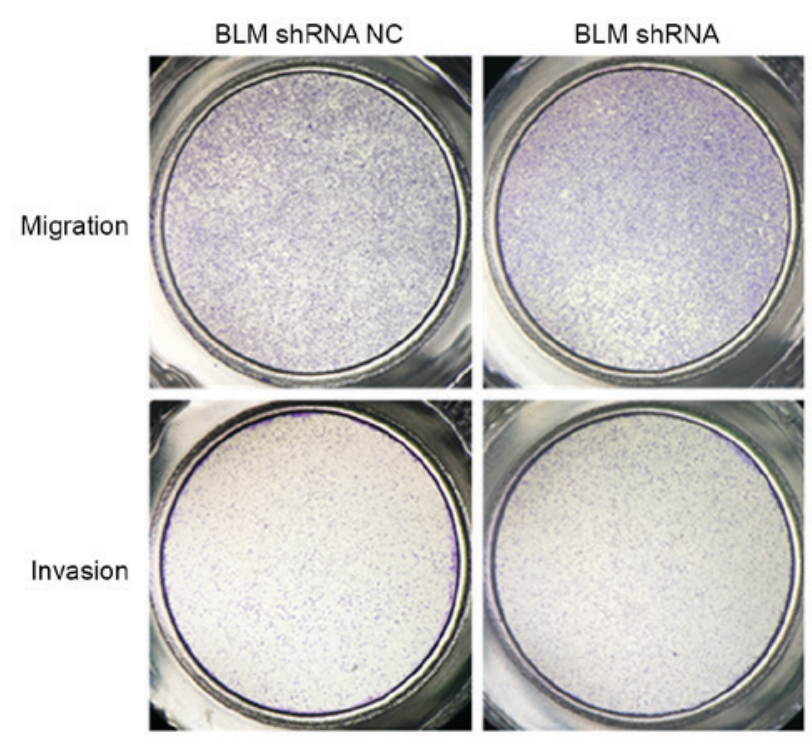

B
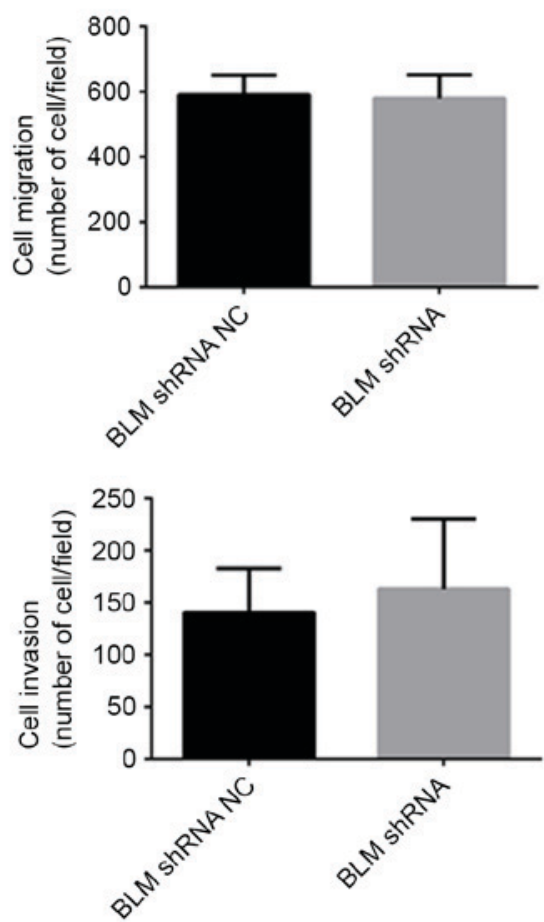

Figure 7. Knockdown of BLM have no effect on PC3 cell migration and invasion. (A) Representative images of cell migration and invasion were detected in PC 3 cells by transwell; (B) Quantification of cell number indicates that cells transfected with BLM shRNA could not up- or down-regulate the cell migration and invasion compared with the $\mathrm{NC}$ group, $\mathrm{n}=3$ per group. BLM, bloom syndrome protein; $\mathrm{NC}$, negative control.

is characterized by proportional dwarfism, sun-sensitive facial erythema, skin pigmentation abnormalities, immunodeficiency and infertility (17). In addition, mutations in BLM are pathogenic due to an increased predisposition for development of many tumor types, including lymphoid and epithelial-derived tumors $(18,19)$. Balci et al $(20)$ reported that colon carcinoma was diagnosed in $\sim 12 \%$ of patients with Bloom syndrome, and Thompson et al (8) reported that heterozygous truncating mutations in BLM increase the risk of breast cancer.

Cell proliferation and cell cycle control are the major regulatory mechanisms of cell growth. A large body of evidence has established the mechanisms of how BLM mutations affect tumor development, including changes to the regulation of many cellular DNA metabolic processes, including DNA replication, DNA repair, telomere maintenance and RNA transcription. BLM expression is high in the S-phase of the cell cycle (21), but BLM depletion has been reported to suppress cell proliferation in human fibroblasts, and BLM-deficient cells have defective S-phase progression (22). Other studies also report that BLM plays a role in recovery from $S$-phase arrest in response to hydroxyurea. This may due to interaction of BLM and p53-binding protein 1 in a Chk1-mediated pathway (23). Our study demonstrated that knockdown of BLM in PC3 cells suppressed cell proliferation and increased cell S-phase arrest. Apoptosis has an important role in tumor progression, Chester et al (24) reported that increased apoptosis occurred in BLM mutant embryos, Kaneko et al (25) suggested that BLM-deficient cells have abnormal regulation of $\mathrm{p} 53$ protein expression and are highly susceptible to apoptosis. This previous study also suggested that p53 mediates cell death by 
inducing mitochondria-related apoptosis (25). Consistently, the current study also revealed that BLM deficiency enhanced cell apoptosis. Taken together, these results provide strong evidence that BLM may play an important role in the genomic stability and DNA repair of PCa cells. Therefore, it is tempting to speculate that BLM could be a useful therapeutic target for treating PCa cases that are susceptible to specific DNA-damaging chemotherapeutic agents.

However, in the present study, silencing of BLM expression had no impact on invasion and migration of PC3 cells. This effect of BLM during tumorigenesis predominantly involves changes in DNA replication and repair through increased fragility of hotspots after replication fork stalling. Without functional BLM, inefficient resolution of DNA linkages at fragile sites gives rise to increased numbers of anaphase ultra-fine DNA bridges and fragile site loci that contribute to chromosomal instability and tumorigenesis (26). However, as indicated by the results of our study, loss of BLM function may not influence the invasion and migration potential of PCa cells.

In summary, our results provide a new perspective for $\mathrm{PCa}$ tumorigenesis and development. These findings highlight a novel phenomenon in the regulation of $\mathrm{PCa}$ progression, which is useful for understanding the effect of BLM on proliferation and apoptosis of PCa cells. Thus, BLM could be a promising biomarker and/or a therapeutic target for PCa treatment. However, further work is necessary to explore the regulatory mechanisms of BLM in PCa.

\section{Acknowledgements}

This work was supported by Beijing Municipal Administration of Hospitals Clinical Medicine Development of Special Funding Support (grant no. ZYLX201408), China Postdoctoral Science Foundation (grant no. 2015M581131).

\section{References}

1. Siegel RL, Miller KD and Jemal A: Cancer statistics, 2015. CA Cancer J Clin 65: 5-29, 2015.

2. Melichar B: PSA, PCA3 and the phi losophy of prostate cancer management. Clin Chem Lab Med 51: 707-712, 2013.

3. Elabbady AA and Khedr MM: Extended 12-core prostate biopsy increases both the detection of prostate cancer and the accuracy of Gleason score. Eur Urol 49: 49-53, 2006.

4. Green L: Digital rectal examination screening for prostate cancer. JAMA 270: 1315, 1993.

5. Ghai S and Toi A: Role of transrectal ultrasonography in prostate cancer. Radiol Clin North Am 50: 1061-1073, 2012.

6. Mohaghegh P, Karow JK, Brosh RM Jr, Bohr VA and Hickson ID: The Bloom's and Werner's syndrome proteins are DNA structure-specific helicases. Nucleic Acids Res 29: 2843-2849, 2001.

7. Böhm S and Bernstein KA: The role of post-translational modifications in fine-tuning BLM helicase function during DNA repair. DNA Repair (Amst) 22: 123-132, 2014.

8. Thompson ER, Doyle MA, Ryland GL, Rowley SM, Choong DY, Tothill RW, Thorne H; kConFab, Barnes DR, Li J, et al: Exome sequencing identifies rare deleterious mutations in DNA repair genes FANCC and BLM as potential breast cancer susceptibility alleles. PLoS Genet 8: e1002894, 2012.
9. de Voer RM, Hahn MM, Mensenkamp AR, Hoischen A, Gilissen C, Henkes A, Spruijt L, van Zelst-Stams WA, Kets CM, Verwiel ET, et al: Deleterious germline BLM mutations and the risk for early-onset colorectal cancer. Sci Rep 5: 14060, 2015.

10. Davari P, Hebert JL, Albertson DG, Huey B, Roy R, Mancianti ML, Horvai AE, McDaniel LD, Schultz RA and Epstein EH Jr: Loss of Blm enhances basal cell carcinoma and rhabdomyosarcoma tumorigenesis in Ptch1+/-mice. Carcinogenesis 31: 968-973, 2010.

11. Johnson AM, Zuhlke KA, Plotts C, McDonnell SK, Middha S, Riska SM, Schaid DJ, Thibodeau SN, Douglas JA and Cooney KA: Mutational landscape of candidate genes in familial prostate cancer. Prostate 74: 1371-1378, 2014.

12. Kämpjärvi K, Kim NH, Keskitalo $\mathrm{S}$, Clark $\mathrm{AD}$, von Nandelstadh P, Turunen M, Heikkinen T, Park MJ, Mäkinen N, Kivinummi K, et al: Somatic MED12 mutations in prostate cancer and uterine leiomyomas promote tumorigenesis through distinct mechanisms. Prostate 76: 22-31, 2016.

13. Ewing CM, Ray AM, Lange EM, Zuhlke KA, Robbins CM, Tembe WD, Wiley KE, Isaacs SD, Johng D, Wang Y, et al: Germline mutations in HOXB13 and prostate-cancer risk. N Engl J Med 366: 141-149, 2012.

14. Douglas JA, Levin AM, Zuhlke KA, Ray AM, Johnson GR, Lange EM, Wood DP and Cooney KA: Common variation in the BRCA1 gene and prostate cancer risk. Cancer Epidemiol Biomarkers Prev 16: 1510-1516, 2007.

15. Agalliu I, Kwon EM, Zadory D, McIntosh L, Thompson J, Stanford JL and Ostrander EA: Germline mutations in the BRCA2 gene and susceptibility to hereditary prostate cancer. Clin Cancer Res 13: 839-843, 2007.

16. Kitano K: Structural mechanisms of human RecQ helicases WRN and BLM. Front Genet 5: 366, 2014.

17. Ellis NA and German J: Molecular genetics of Bloom's syndrome. Hum Mol Genet 5: 1457-1463, 1996.

18. Schuetz JM, MaCarthur AC, Leach S, Lai AS, Gallagher RP, Connors JM, Gascoyne RD, Spinelli JJ and Brooks-Wilson AR: Genetic variation in the NBS1, MRE11, RAD50 and BLM genes and susceptibility to non-Hodgkin lymphoma. BMC Med Genet 10: 117, 2009.

19. Goss KH, Risinger MA, Kordich JJ, Sanz MM, Straughen JE, Slovek LE, Capobianco AJ, German J, Boivin GP and Groden J: Enhanced tumor formation in mice heterozygous for Blm mutation. Science 297: 2051-2053, 2002.

20. Balc1 S and Aktas D: Mucinous carcinoma of the colon in a 16-year-old Turkish boy with Bloom syndrome: Cytogenetic, histopathologic, TP53 gene and protein expression studies. Cancer Genet Cytogenet 111: 45-48, 1999.

21. Singh DK, Popuri V, Kulikowicz T, Shevelev I, Ghosh AK, Ramamoorthy M, Rossi ML, Janscak P, Croteau DL and Bohr VA: The human RecQ helicases BLM and RECQL4 cooperate to preserve genome stability. Nucleic Acids Res 40: 6632-6648, 2012.

22. Park SJ, Lee YJ, Beck BD and Lee SH: A positive involvement of RecQL4 in UV-induced S-phase arrest. DNA Cell Biol 25: 696-703, 2006.

23. Sengupta S, Robles AI, Linke SP, Sinogeeva NI, Zhang R, Pedeux R, Ward IM, Celeste A, Nussenzweig A, Chen J, et al: Functional interaction between BLM helicase and 53BP1 in a Chk1-mediated pathway during S-phase arrest. J Cell Biol 166: 801-813, 2004.

24. Chester N, Kuo F, Kozak C, O'Hara CD and Leder P: Stage-specific apoptosis, developmental delay, and embryonic lethality in mice homozygous for a targeted disruption in the murine Bloom's syndrome gene. Genes Dev 12: 3382-3393, 1998.

25. Kaneko H, Fukao T, Kasahara K, Yamada T and Kondo N: Augmented cell death with Bloom syndrome helicase deficiency. Mol Med Rep 4: 607-609, 2011.

26. Chan KL, Palmai-Pallag T, Ying S and Hickson ID: Replication stress induces sister-chromatid bridging at fragile site loci in mitosis. Nature Cell Biol 11: 753-760, 2009. 